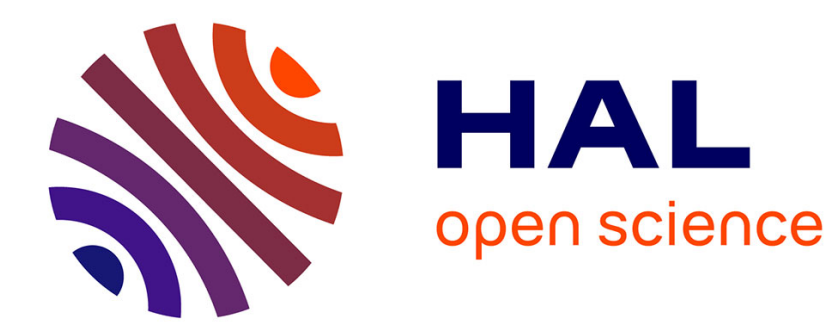

\title{
Schéma de niveaux de 223Ra II. Spectre d'électrons de conversion interne émis dans la désintégration $227 \mathrm{Th} \rightarrow$ 223Ra
}

\author{
C. Briançon, C. Vieu
}

\section{- To cite this version:}

C. Briançon, C. Vieu. Schéma de niveaux de 223Ra II. Spectre d'électrons de conversion interne émis dans la désintégration 227Th $\rightarrow$ 223Ra. Journal de Physique, 1971, 32 (5-6), pp.373-380. 10.1051/jphys:01971003205-6037300 . jpa-00207088

\section{HAL Id: jpa-00207088 https://hal.science/jpa-00207088}

Submitted on 1 Jan 1971

HAL is a multi-disciplinary open access archive for the deposit and dissemination of scientific research documents, whether they are published or not. The documents may come from teaching and research institutions in France or abroad, or from public or private research centers.
L'archive ouverte pluridisciplinaire HAL, est destinée au dépôt et à la diffusion de documents scientifiques de niveau recherche, publiés ou non, émanant des établissements d'enseignement et de recherche français ou étrangers, des laboratoires publics ou privés. 
Classification

Physics Abstracts

$12.17,12.20$

\title{
SCHÉMA DE NIVEAUX DE ${ }^{223}$ Ra
}

\section{SPECTRE D'ÉLECTRONS DE CONVERSION INTERNE ÉMIS DANS LA DÉSINTÉGRATION ${ }^{227}$ Th $\rightarrow{ }^{223}$ Ra}

\author{
C. BRIANÇON et C. VIEU
}

Centre de Spectrométrie Nucléaire et de Spectrométrie de Masse du C. N. R. S., Orsay, France

(Reçu le 26 janvier 1971)

\begin{abstract}
Résumé. - Cet article expose l'ensemble de nos études sur les quelques 300 raies de conversion du spectre d'électrons émis dans la désintégration $227 \mathrm{Th} \rightarrow{ }^{223} \mathrm{Ra}$ : étude par spectrométrie à focalisation semi-circulaire et à double focalisation, ce qui a permis de fixer toutes les intensités en valeur absolue par désintégration $\alpha$ et de fournir un grand nombre de multipolarités.
\end{abstract}

Abstract. - This paper summarizes all our results concerning the complex conversion spectrum emitted after the ${ }^{227 \mathrm{Th}} \rightarrow{ }^{223} \mathrm{Ra}$ decay. Semi-circular magnetic spectrography and absolute measurements with a double focussing spectrometer have yielded absolute intensities in $\alpha$ desintegration units, as well as many multipolarities.

Dans le cadre de notre étude, par différentes méthodes, du schéma de niveaux de ${ }^{223} \mathrm{Ra}$, noyau à la limite de la zone de grande déformation (Voir la $1^{\text {re }}$ partie concernant la spectrométrie $\gamma$ directe [1] ainsi que la référence [2] qui contiennent une bibliographie complète), nous donnons ici l'ensemble de nos résultats acquis en spectrométrie de conversion au cours des dernières années. Ce spectre, très complexe puisqu'il comporte plus de 300 raies au-dessous de $400 \mathrm{keV}$, avait fait l'objet de mesures préalables au spectrographe à focalisation semi-circulaire et à détection photographique, méthode particulièrement bien adaptée au cas présent de spectre très riche en raies [1] [2]. Ces mesures ont été reprises par une méthode permettant d'obtenir les intensités des raies de conversion en valeur absolue avec le spectrographe à double focalisation $\pi \sqrt{2}$ du Centre, avec détermination simultanée des intensités $\alpha$ absolues des sources.

En ce qui concerne la spectrométrie à focalisation semi-circulaire, l'étude a été effectuée au moyen de 11 sources (de 0,1 à $2 \mathrm{mCi}$ ) obtenues à partir de la volatilisation de l'oxyde de ${ }^{227} \mathrm{Th}$ dont la préparation a déjà été décrite dans la référence [1]. Un grand nombre de spectres a dû être enregistré (plus de 100) et ce nombre élevé s'explique par :

a) La gamme d'énergie explorée (0 à $400 \mathrm{keV}$ ) et la grande densité de raies, ce qui a nécessité l'utilisation de plusieurs spectrographes à des champs de 30 , 60,100 et 150 gauss. b) La faible gamme d'intensité pour laquelle une émulsion reste suffisamment linéaire, alors que dans ce spectre les intensités des raies observées varient dans des rapports pouvant aller jusqu'à $2 \times 10^{3}$.

c) La nécessité d'utiliser une préaccélération des électrons (10 à $15 \mathrm{kV}$ ) pour les faibles énergies.

d) La variété des supports de source employés :

- pour la région des basses énergies, supports très minces (feuilles d'oxyde d'aluminium) qui donnent un fond photographique moindre et une meilleure résolution pour les raies, mais au prix d'une efficacité plus faible.

- pour la région des sur «tranche » de $2 / 10 \mathrm{~mm}$ d'aluminium, donnant des raies fines mais présentant plus de queue vers les basses énergies. Ces sources étaient recouvertes ou non d'une dizaine de $\mu \mathrm{g} / \mathrm{cm}^{2}$ d'aluminium si l'on voulait supprimer les reculs, donc les bandes Doppler du côté des hautes énergies des raies.

Enfin, les poses étant rarement courtes, il y a accumulation rapide des dérivés de ${ }^{223} \mathrm{Ra}$, dont il a fallu tenir compte au moyen de poses successives permettant de contrôler l'origine des raies par l'évolution de leur intensité en fonction du temps.

Le spectre étant impossible à représenter dans sa totalité nous donnons deux exemples de microdensitogrammes à basse énergie (Fig. $1 a$ et $1 b$ ) et moyenne énergie (Fig. $2 a$ et $2 b$ ). 
Par ailleurs deux séries de mesures au spectromètre $\pi \sqrt{2}$ à l'aide de sources intenses $(6 \mathrm{mCi}$ déposés sur des tranches de $7 / 10 \mathrm{~mm}$ d'aluminium) jalonnent le spectre de 150 raies permettant ainsi d'étalonner en valeur absolue l'ensemble du spectre de conversion. La détection des électrons a été réalisée à l'aide d'un compteur à fenêtre mince rempli d'un mélange argonpropane à la pression de $10 \mathrm{~cm}$ de mercure. Le temps mort du compteur a été réduit à $5 \times 10^{-6} \mathrm{~s}$ à l'aide d'un circuit électronique limitant l'extension de la décharge Geiger. Cet ensemble de détection, pouvant compter jusqu'à $5 \times 10^{3}$ événements par seconde sans risque d'atteindre la saturation du compteur, a permis de réduire considérablement les temps de mesure avec des sources de grande activité, de manière à minimiser l'importance des dérivés dont l'accumulation est rapide, la période de décroissance de ${ }^{223} \mathrm{Ra}$ étant de 11,2 jours (exemples de spectres : Fig. $3 a, b, c, d)$.

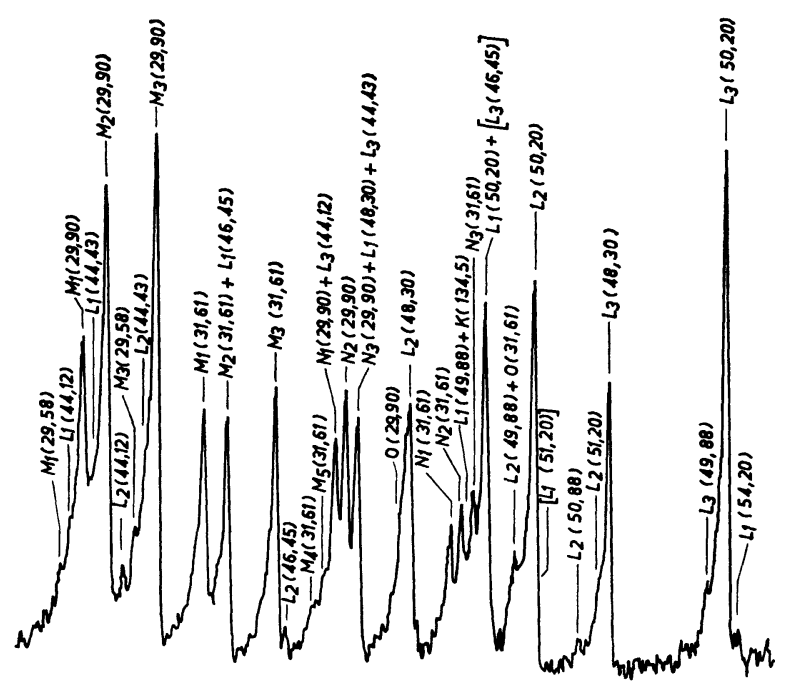

(a)

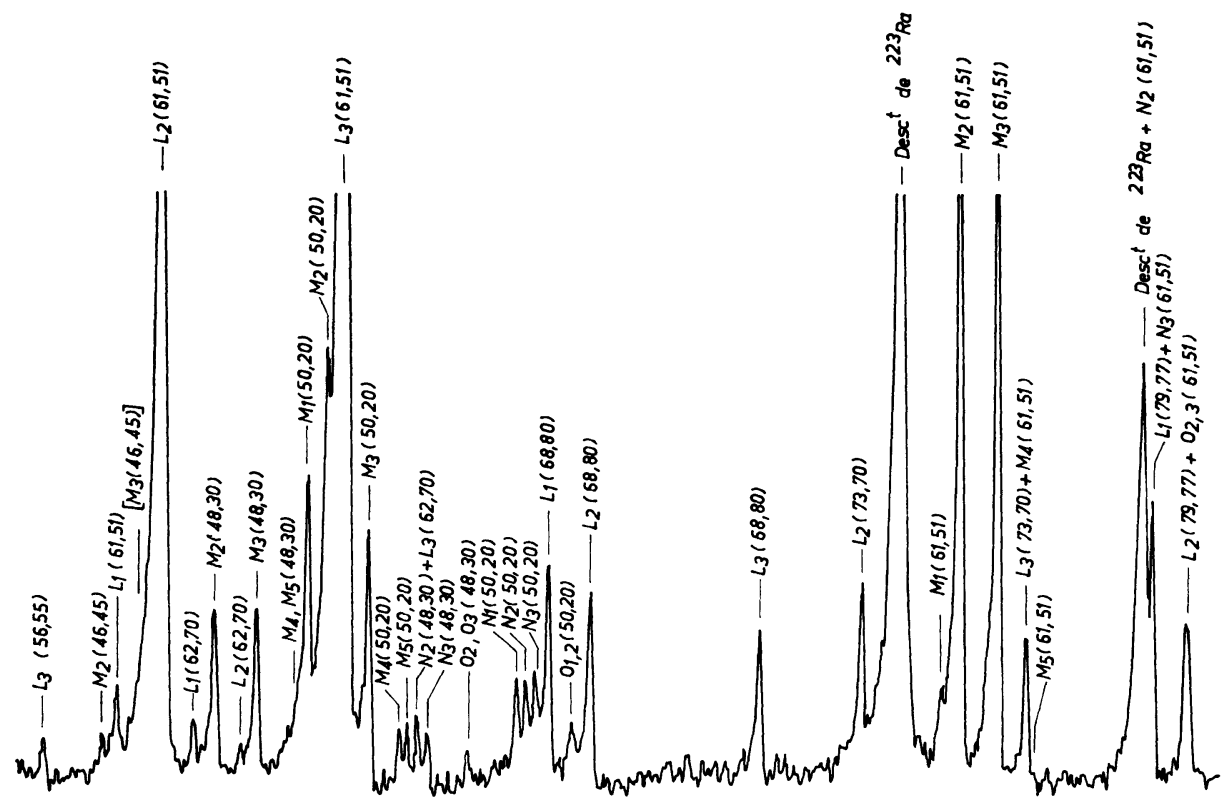

(b)

FIG. $1 a$ et $b$. - Microdensitogrammes du spectre des électrons de conversion de basse énergie émis lors de la désexcitation de ${ }^{223} \mathrm{Ra}$.

Le tableau 1 présente l'ensemble des résultats obtenus, avec l'énergie de la transition, les raies de conversion observées, les intensités de conversion dans les couches $\mathrm{K}$ et $\mathrm{L}$, exprimées en unité de désintégration $\alpha$, et qui ont servi à déterminer les multipolarités. Dans la deuxième colonne, les raies complexes ont été indiquées entre parenthèses, le détail de cette complexité étant donné dans la référence [2].

Nous n'avons pas pu donner dans ce tableau toutes les erreurs concernant les intensités : d'une façon générale, on peut affecter d'une erreur d'environ $6 \%$ les raies les plus intenses du spectre (de $2 \times 10^{-2}$ à $10^{-1}$ ), d'environ $10 \%$ les lignes d'intensités comprises entre $2 \times 10^{-4}$ et $2 \times 10^{-2}$, de $20 \%$ les intensités jusqu'à $5 \times 10^{-5}$, les erreurs pouvant aller jusqu'à $40 \%$ pour les intensités plus faibles, tout ceci bien sûr pour des raies ne provenant pas de décomposition. Dans ce dernier cas, une erreur supplémentaire vient s'ajouter dans chaque cas spécifique, la complexité des raies étant mentionnée dans la deuxième colonne du tableau.

Cependant, les rapports d'intensités des raies, utilisés dans la détermination des multipolarités, sont affectés d'erreurs moindres.

En ce qui concerne les énergies, la précision est meilleure que $1 \%$. Par ailleurs, nous n'avons pas indiqué ici les attributions des transitions car celles-ci sont fixées par l'ensemble des méthodes utilisées pour l'étude de ce noyau et seront donc données globalement dans l'article suivant (III). 
TABleau I

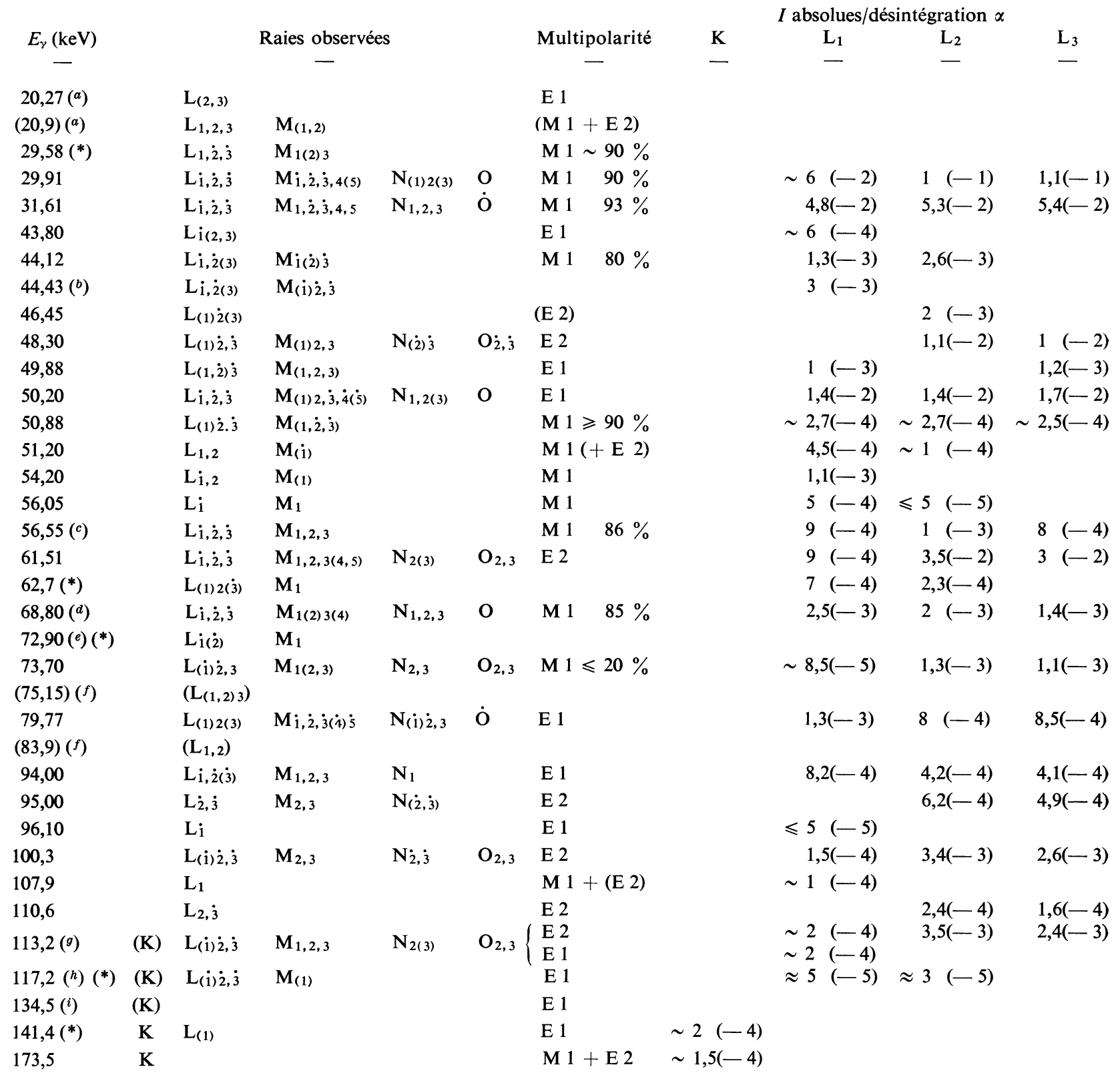

(•) Toutes les raies de conversion ont été observées par spectrographie semi-circulaire ; celles surmontées d'un point ont été également mesurées au spectrographe $\pi \sqrt{ } 2$ et ont fixé l'échelle des intensités absolues.

$\left(^{*}\right)$ Le signe $(*)$ indique que les problèmes soulevés par ces transitions ont été tranchés au moyen des coïncidences $\gamma-\gamma$ (Voir article III).

(a) Les raies $\mathrm{L}$ de ce rayonnement ont été obtenues en appliquant une tension d'accélération aux électrons. Les intensités ne sont pas données, car dans cette région d'énergie très faible l'imprécision est trop grande.

${ }^{(b)}$ Ce rayonnement est double, désexcitant les deux niveaux de parités opposées situés à $174,7 \mathrm{keV}$ (Voir note $\left(^{g}\right)$ ).

(c) L intensité de transition trouvée à partir des électrons de conversion est en contradiction avec celle trouvée par coïncidences $\gamma-\gamma$ (Voir article III).

(d) Les coïncidences $\gamma-\gamma$ montrent qu'il existe un autre rayonnement de $68,7 \mathrm{keV}$, mais de multipolarité E 1, et qui n'affecte pas les raies intenses de conversion du rayonnement de $68,80 \mathrm{keV}$.

(e) Rayonnement dédoublé en deux composantes (E 1 et $\mathrm{M} 1+\mathrm{E} 2)$ alimentant les deux niveaux de parités opposées situés à $174,7 \mathrm{keV}$ (Voir note $\left({ }^{a}\right)$ ).

$(f)$ Cette transition est possible, mais les intensités de conversion sont trop imprécises pour déterminer la multipolarité.

$\left.{ }^{g}\right)$ Ce rayonnement a été trouvé double (2 rayonnements de nature E 1 et $\mathrm{E} 2$ respectivement) de par la contradiction entre intensités $\gamma$ et d'électrons. Par ailleurs les coïncidences $\alpha-\gamma$ et $\gamma-\gamma$ ont vérifié que ces deux rayonnements désexcitent deux niveaux (de parités opposées), très proches en énergie à $174,7 \mathrm{keV}$ (les raies $\mathrm{L}_{2}$ et $\mathrm{L}_{3}$ du rayonnement $\mathrm{E} 1$ sont entièrement masquées par celles, très intenses, du rayonnement $\mathrm{E} 2$ ).

${ }^{(h)}$ Ce rayonnement est double, d'après les coïncidences $\gamma-\gamma$ (Voir article III). Tous deux sont de multipolarité E 1.

(i) Raie $\mathrm{K}$ masquée par $\mathrm{L}_{1}(49,88)$; l'absence des raies de conversion $\mathrm{L}$ permet d'attribuer la multipolarité $\mathrm{E} 1$ à ce rayonnement. 


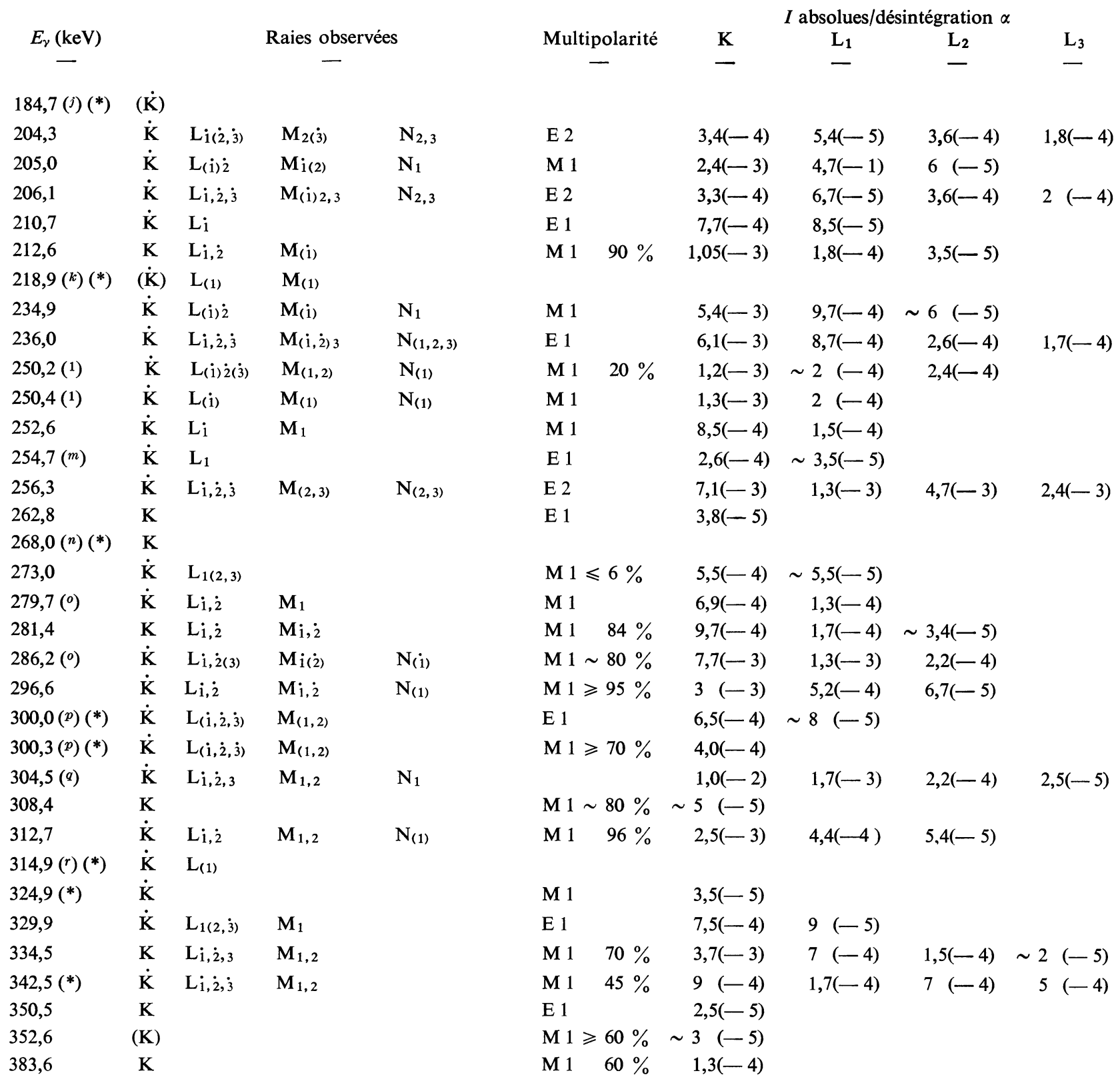

${ }^{(j)}$ Transition complexe pour laquelle les énergies obtenues par spectrométrie $\gamma$ et électrons ne sont pas identiques (Voir article III).

$\left({ }^{k}\right)$ Rayonnement double d'après les coïncidences $\gamma-\gamma$ (rayonnements de 218,8 keV (M 1) et 219,0 keV (E 1)): les raies de conversion du rayonnement de $218,8 \mathrm{keV}$ sont prédominantes (Voir article III).

$(l)$ Les raies $\mathrm{K}$ de ces deux rayonnements sont séparées, mais non les raies L. L'intensité $\gamma$ est départagée par coïncidences, d'où les multipolarités et les intensités données pour les raies L.

$\left.{ }^{m}\right)$ Intensité $\gamma$ et multipolarité sont déduites des coïncidences $\gamma-\gamma$.

(n) Rayonnement double, voir coïncidences $\gamma-\gamma$ (article III).

$\left(^{o}\right)$ Ce rayonnement présente une anomalie de conversion (voir paragraphe suivant).

$(p)$ Les intensités $\gamma$ et multipolarités sont déduites des coïncidences $\gamma-\gamma$.

$\left({ }^{q}\right)$ Voir le paragraphe suivant $\left({ }^{c}\right)$ pour la multipolarité de ce rayonnement.

$(r)$ Deux rayonnements sont superposés d'énergies 314,8 keV (M $1 \geqslant 93 \%$ ) et 314,9 keV (E 1) (Voir coïncidences $\gamma-\gamma)$.

Cas de transitions anormales du point de vue de la conversion : effets de pénétration des électrons dans le volume nucléaire. - Pour quelques transitions, à caractère prédominant $\mathrm{M} 1$, il a été impossible d'obtenir un accord avec les différentes tables de coefficients de conversion théoriques, dans la limite des barres d'erreur : il s'agit de rayonnements qui présentent des facteurs de ralentissement M 1 plus élevés que les autres (article III). Nous discuterons ici plus en détail le cas des rayonnements de $279,7,286,2$ et $304,5 \mathrm{keV}$ qui possèdent des lignes $\gamma$ et d'électrons suffisamment intenses pour être mesurées avec précision. 


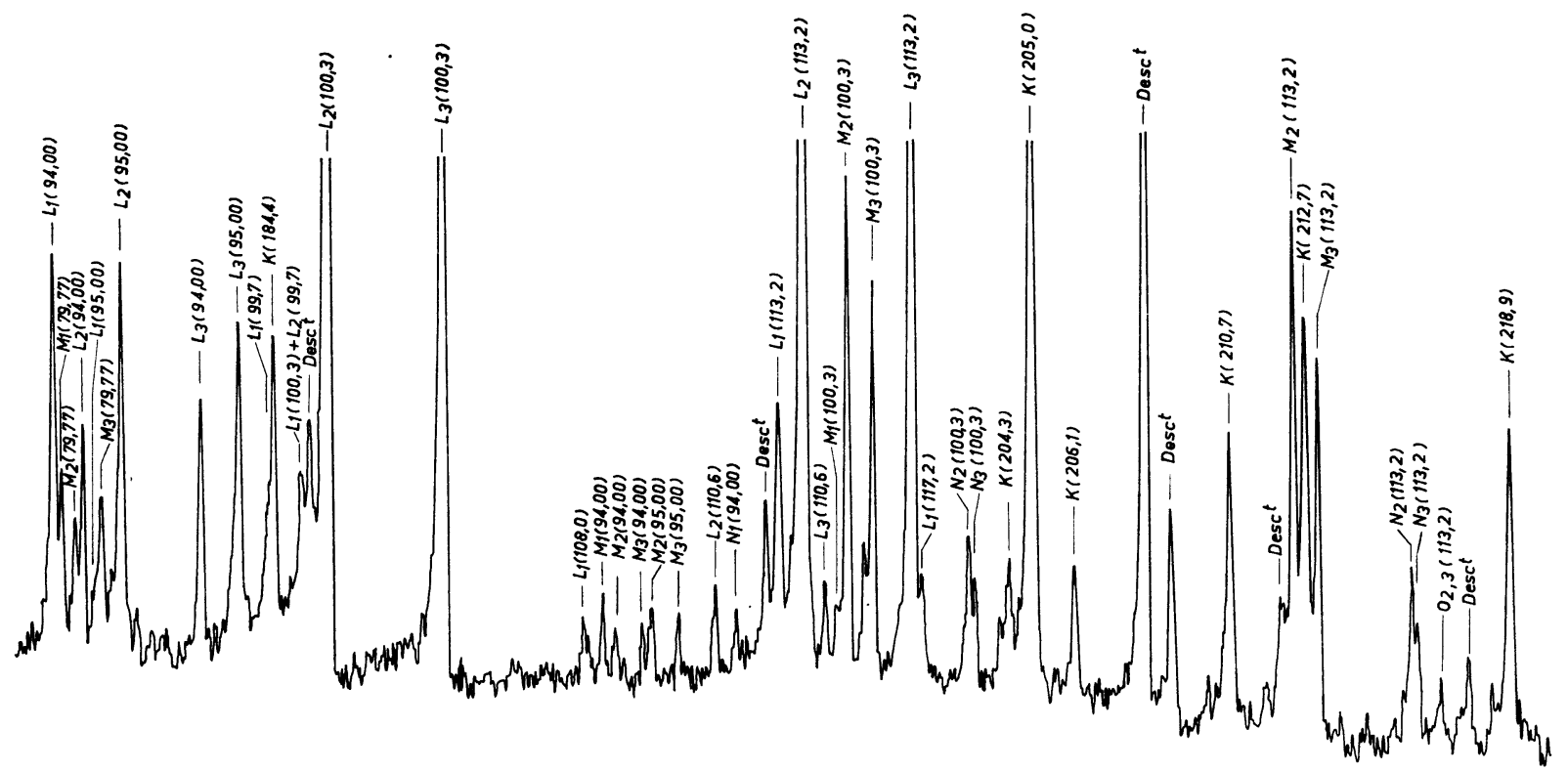

(a)

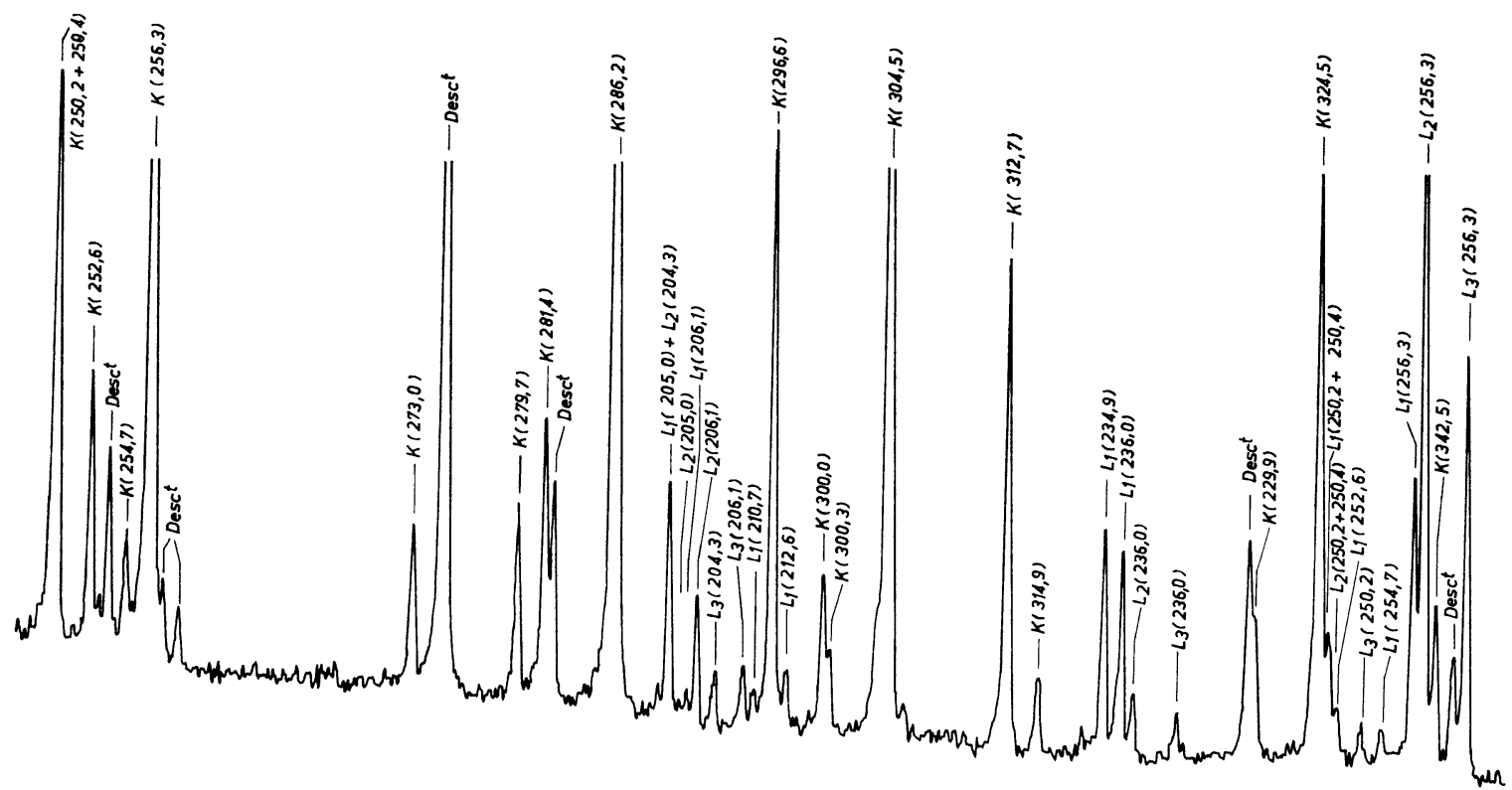

(b)

Fig. $2 a$ et $b .-$ Microdensitogrammes du spectre de conversion à moyenne énergie (seules les raies $\mathrm{K}$ et $\mathrm{L}$ sont mentionnées).

a) RAyonnement DE $286,2 \mathrm{keV}$. - Le tableau II que l'on obtient à partir des coefficients de conversion montre les valeurs du taux de mélange $\delta^{2}(\mathrm{E} 2 / \mathrm{M} 1)$ expérimentaux :

\section{TABLEAU II}

$\alpha$ théor.

(Sliv [3])

\begin{tabular}{|c|c|c|c|c|c|c|}
\hline \multirow[b]{2}{*}{ Raie } & \multirow[b]{2}{*}{$I_{\mathrm{e}-}$} & \multirow{2}{*}{\multicolumn{2}{|c|}{$\alpha_{\exp }$}} & \multicolumn{2}{|c|}{ (Sliv [3]) } & \multirow{2}{*}{$\delta^{2}\left(\frac{\mathrm{E} \mathrm{2}}{\mathrm{M} 1}\right)$} \\
\hline & & & & M 1 & E 2 & \\
\hline - & - & & - & & & - \\
\hline $\mathrm{K}$ & $(7,7 \pm 0,5) \quad 10^{-3}$ & 0,51 & $\pm 0,03$ & 0,65 & 0,075 & $0,32 \pm 0,06$ \\
\hline $\mathrm{L}_{1}$ & $(1,33 \pm 0,08) 10^{-3}$ & 0,089 & $\pm 0,005$ & 0,11 & 0,014 & $0,28 \pm 0,08$ \\
\hline $\mathrm{L}_{2}$ & $(2,15 \pm 0,2) \quad 10^{-4}$ & 0,0144 & $4 \pm 0,0015$ & 0,013 & 0,043 & $0,05 \pm 0,02$ \\
\hline $\mathrm{L}_{1} / \mathrm{L}_{2}$ & & 6,2 & $\pm 0,4$ & 8,5 & 0,33 & $0,12 \pm 0,03$ \\
\hline
\end{tabular}




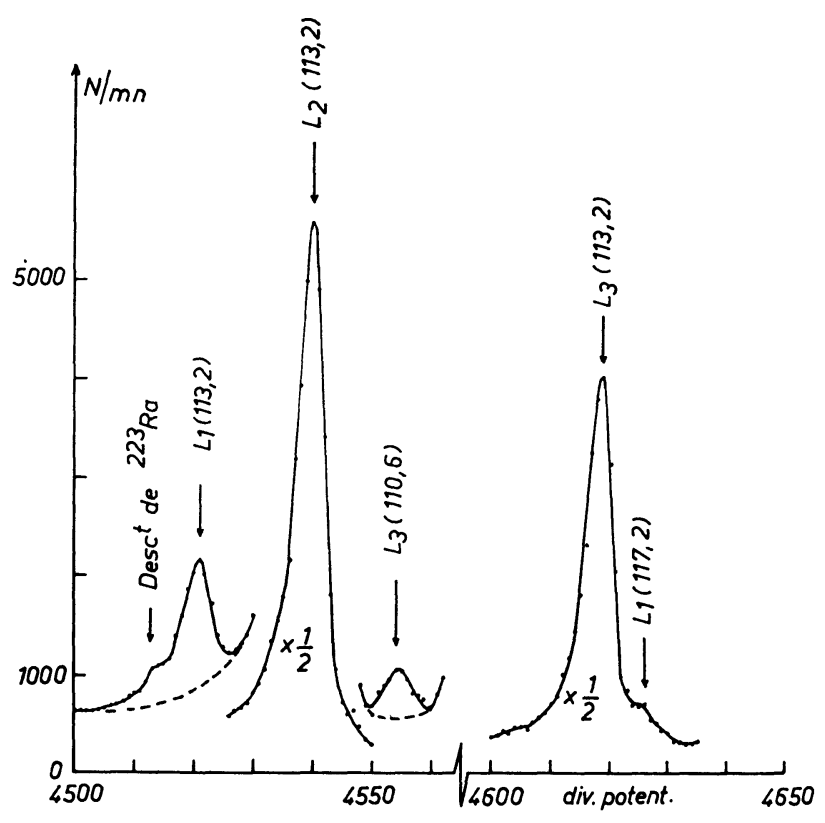

(a)

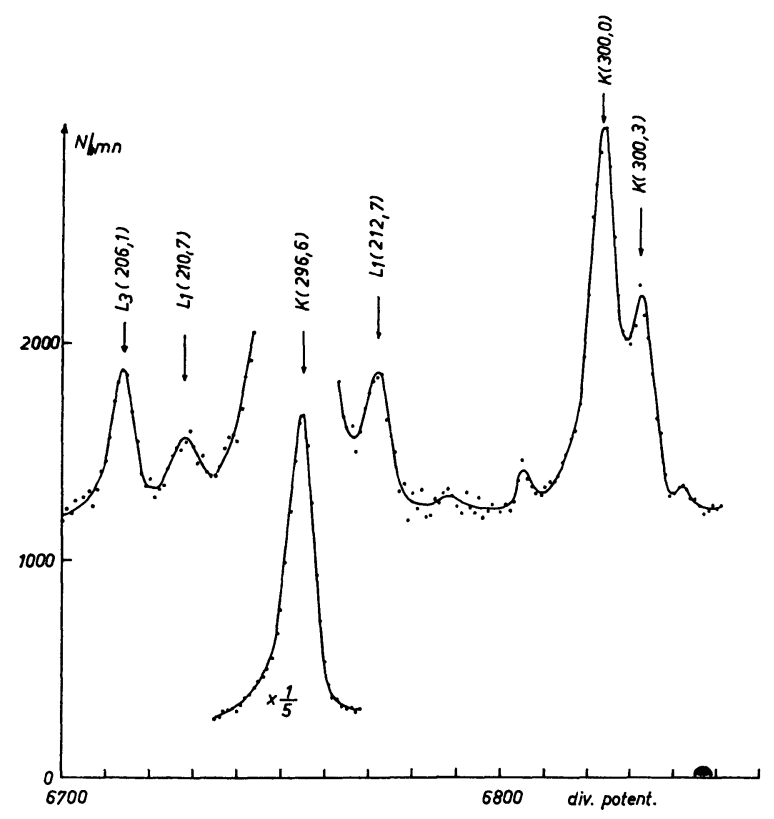

(c)

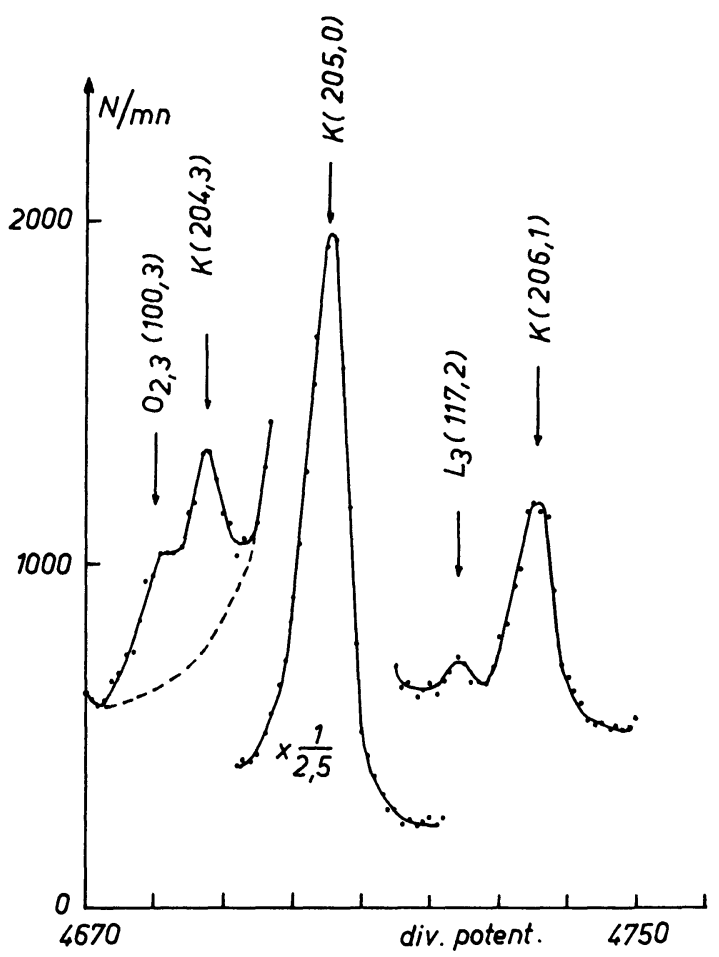

(b)

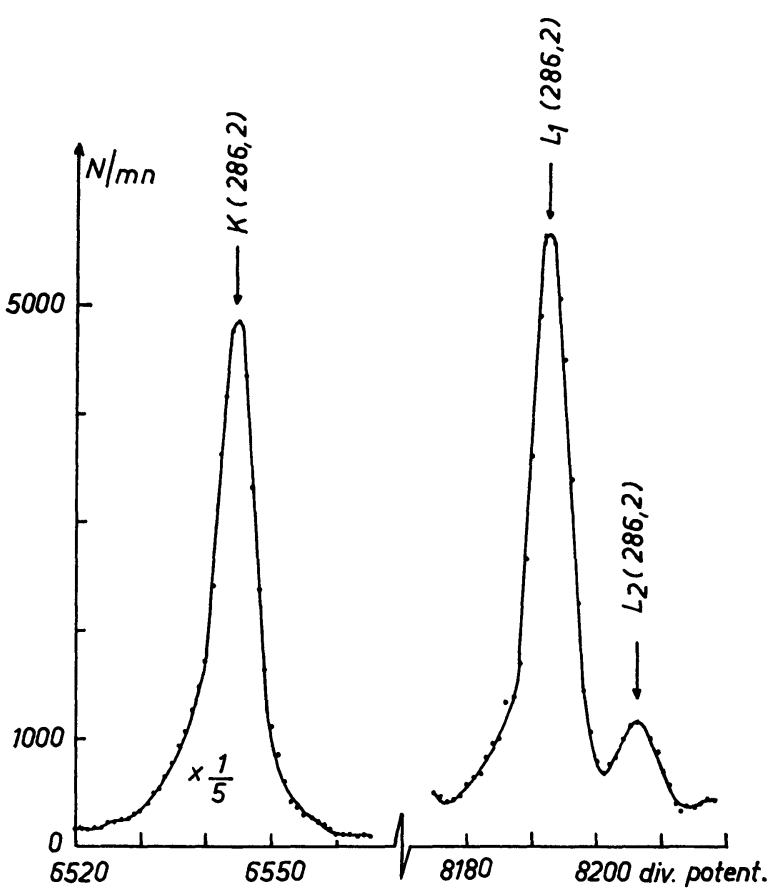

(d)

FIG. $3 a, b, c, d$. - Exemples de raies de conversion obtenues à l'aide d'un spectrographe $\pi \sqrt{2}$.

Les écarts des valeurs de $\delta^{2}$ ainsi obtenues, incompatibles avec les barres d'erreur, nous ont conduit à envisager une anomalie de la conversion $\mathrm{M} 1$ et à calculer le facteur $\lambda$, rapport de l'élément de matrice de pénétration à l'élément de matrice de l'émission $\gamma$, à partir de l'expression des coefficients de conversion donnée par Church et Weneser [4], [5] ; par exemple, pour la couche $\mathrm{K}$ :

$$
\begin{array}{r}
\alpha_{\mathrm{M} 1}^{\mathrm{K}}(\lambda)=\alpha_{\mathrm{M} 1}^{\mathrm{K} \rightarrow \mathrm{L}-1 / 2}(\text { Sliv })\left|1-C_{\mathrm{L}-1 / 2}(\mathrm{R})(\lambda-1)\right|^{2}+ \\
+\alpha_{\mathrm{M} 1}^{\mathrm{K} \rightarrow \mathrm{L}+1 / 2} \text { (Sliv) }
\end{array}
$$

où $\mathrm{L} \pm \frac{1}{2}$ décrivent les deux états finaux possibles. 
Le deuxième terme étant inférieur à $10 \%$ du premier, on peut utiliser la formule simplifiée :

$$
\alpha_{M 1}^{\mathrm{K}}(\lambda)=\alpha_{M 1}^{\mathrm{K}} \text { (Sliv) }\left|1-(\lambda-1) C_{\mathrm{K}}\right|^{2}
$$

En utilisant la valeur du coefficient $C_{\mathrm{K}}$ donnée par Church et Weneser [5] et en en déduisant les valeurs $\mathrm{C}_{\mathbf{L}_{1}}$ et $\mathrm{C}_{\mathbf{L}_{2}}$ pour les autres sous-couches suivant Herrlander et Graham [6], la valeur de $\lambda \sim 3,5$ est en accord avec un taux de mélange $\delta^{2}=0,10$ (Fig. 4).

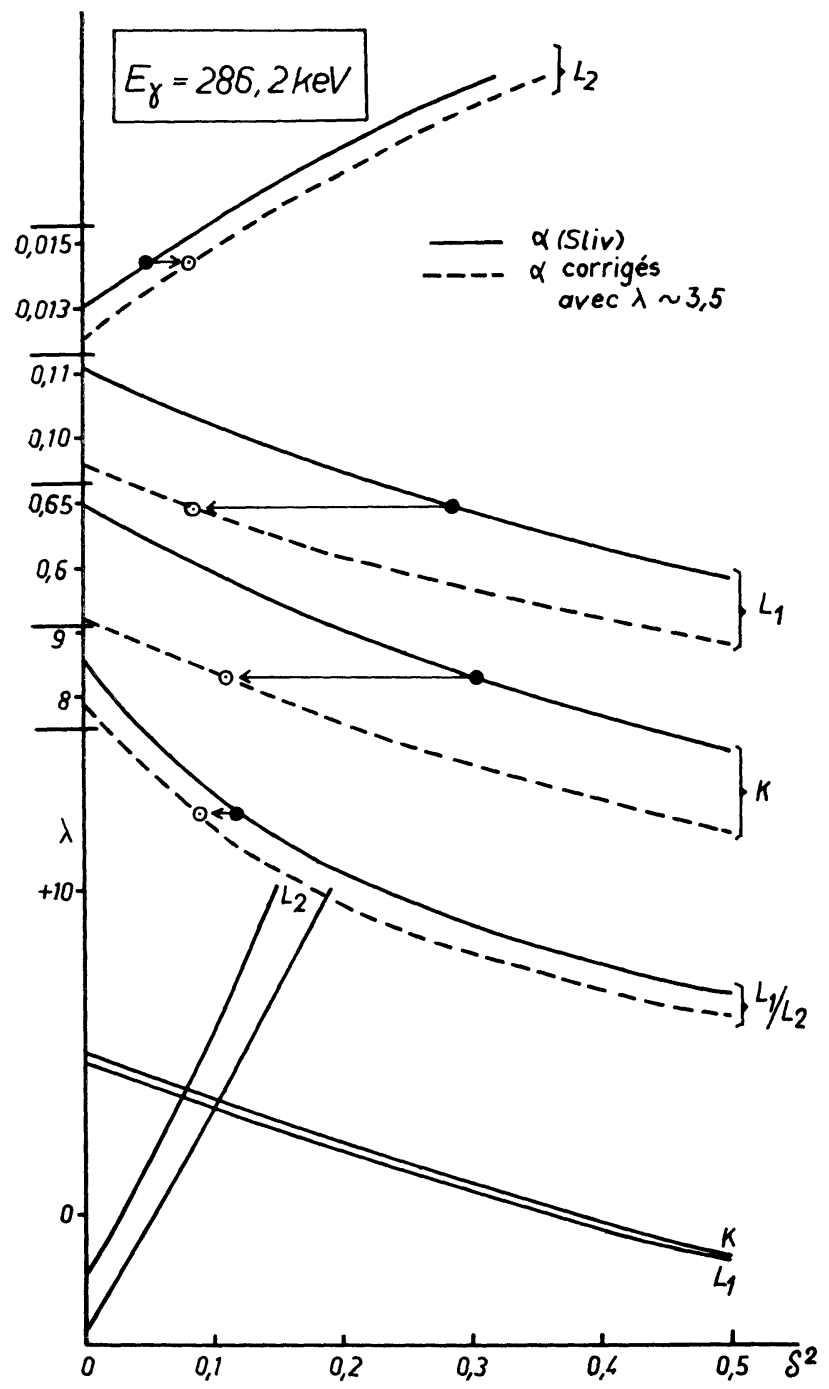

FIG. 4. - Anomalie de la conversion du rayonnement de $286,2 \mathrm{keV}$

Sur cette même figure ont été portés en fonction de $\delta^{2}$ les coefficients de conversion de Sliv, ceux corrigés en prenant $\lambda=3,5$ et en utilisant la formule approchée donnée ci-dessus, ainsi que les points expérimentaux. On peut constater que les effets dynamiques dus à la pénétration des électrons ont bien une influence semblable sur les couches $s\left(K\right.$ et $\left.L_{1}\right)$ et moindre sur la couche $L_{2}\left(2 \mathrm{p} \frac{1}{2}\right)$.

b) Rayonnement de $279,7 \mathrm{keV}$. - Nous ne disposons, pour ce rayonnement, que des intensités absolues de conversion dans les couches $K$ et $L_{1}$ ainsi que de $I_{\gamma}=(6 \pm 1) 10^{-4}$.

\section{TABLEAU III}

\begin{tabular}{cccccc} 
& & & & $\begin{array}{c}\alpha \text { théor. } \\
\text { (Sliv) }\end{array}$ \\
Raie & $I_{\mathrm{e}^{-}}$ & $\alpha_{\text {exp }}$ & M 1 & E 2 \\
- & - & - & \multicolumn{2}{c}{-} \\
$\mathrm{K}$ & $(6,9 \pm 1)$ & $10^{-4}$ & $1,15 \pm 0,2$ & 0,70 & 0,084 \\
$\mathrm{~L}_{1}$ & $(1,3 \pm 0,2)$ & $10^{-4}$ & $0,21 \pm 0,05$ & 0,12 & 0,014
\end{tabular}

Ce rayonnement présente donc une conversion anormalement forte. Une analyse identique à celle décrite pour le rayonnement de $286,2 \mathrm{keV}$, en tenant compte de ce que $I_{\mathrm{e}_{-}}\left(\mathrm{L}_{3}\right)<3 \times 10^{-5}$, permet de fixer une limite supérieure égale à 0,2 pour $\delta^{2}$ avec :

$$
-14<\lambda<-10
$$

ou $83<\lambda<87$, le premier domaine de variation de $\lambda$ étant le plus probable.

c) Rayonnement de $304,5 \mathrm{keV}$. - Pour ce rayonnement, le tableau IV montre la conversion anormale :

TABLEAU IV

\begin{tabular}{lcllccc} 
& & & & \multicolumn{2}{c}{$\alpha$ théor. } \\
(Sliv) \\
Raies & $I_{\mathrm{e}-}$ & & $\alpha_{\exp }$ & $\mathrm{M} \mathrm{1}$ & $\mathrm{E} 2$ \\
- & $\overline{-}$ & & - & - & $-\overline{0}$ \\
$\mathrm{~K}$ & $(1,0 \pm 0,5)$ & $10^{-2}$ & 1,0 & $\pm 0,07$ & 0,57 & 0,068 \\
$\mathrm{~L}_{1}$ & $(1,7 \pm 0,1)$ & $10^{-3}$ & 0,17 & $\pm 0,015$ & 0,096 & 0,012 \\
$\mathrm{~L}_{2}$ & $(2,2 \pm 0,3)$ & $10^{-4}$ & 0,022 & $\pm 0,004$ & 0,011 & 0,034 \\
$\mathrm{~L}_{3}$ & $(2,5 \pm 0,8)$ & $10^{-5}$ & 0,002 & $5 \pm 0,001$ & 0,0006 & 0,015
\end{tabular}

Devant le fait que cette transition a lieu entre deux niveaux de même spin et même parité, comme le montreront nos mesures de corrélations angulaires $\alpha-\gamma$, nous avons envisagé la possibilité d'une proportion de transition $\mathrm{E} 0$. L'analyse la plus générale a donc été effectuée en termes de : proportion de transition $\mathrm{E} 0$ + rayonnement $\mathrm{M} 1$ anormal + proportion de rayonnement E 2. Mais compte tenu des barres d'erreur, cette analyse ne conduit pas à une solution sans ambiguïté pour les raisons suivantes : en fonction du taux de mélange $\delta^{2}(\mathrm{E} 2 / \mathrm{M} 1)$, on trouve toujours une plage d'accord entre les différentes raies de conversion, l'accord étant réalisé pour une série de valeurs liées de $\lambda$ et du taux $z$ de transition E 0. Par exemple :

$$
\begin{array}{ccccc}
z & 0 & 0,1 & 0,2 & 0,3 \\
\bar{\lambda} & -\overline{15} \pm 4 & -\overline{10 \pm 3} & -\overline{5 \pm 2} & +\overline{1} \pm 2
\end{array}
$$

Le taux de mélange $\delta^{2}$ en résultant est de 0,2 à 0,3 , mais nous verrons que ce taux sera en désaccord avec le taux déduit des corrélations angulaires $\alpha-\gamma$ $\left(\delta^{2} \leqslant 0,04\right)$.

Il faut remarquer que l'interprétation ci-dessus est sensible aux simplifications introduites dans les théories des transitions $\mathrm{E} 0$ et des rayonnements $\mathrm{M} 1$ anormaux. C'est pourquoi il sera intéressant de 
tester par une autre méthode, par exemple corrélations $\alpha-\mathrm{e}^{-}$ou $\gamma-\mathrm{e}^{-}$, les taux de mélange de ces différents rayonnements présentant une conversion anormale.

Conclusion. - La présente étude complète les expériences de spectrométrie directe concernant le noyau de ${ }^{223} \mathrm{Ra}$. Mais il est vite apparu que, bien que fournissant beaucoup de résultats, celles-ci ne suffisaient pas à résoudre les problèmes posés par ce schéma de niveaux, ce qui a conduit à recourir à des méthodes d'analyse multidimensionnelle [7], de mesures de vies moyennes et de corrélations angulaires, méthodes dont les résultats seront exposés dans cette série d'articles.

Nous sommes extrêmement reconnaissants envers M. R. J. Walen qui a préparé les sources dont la haute qualité a permis de réaliser ce travail : nous le remercions très sincèrement pour son aide et pour les conseils qu'il nous a apportés au cours de nombreuses discussions, ainsi que M. M. Valadares qui a accompagné ce travail depuis son origine. Nos remerciements vont également à $\mathbf{M}$. J. S. Dionisio pour l'utilisation du spectrographe $\pi \sqrt{2}$ dont il a dirigé la mise au point au Centre.

\section{Bibliographie}

[1] Briançon (Ch.), Walen (R. J.), J. Physique, 1969, 30, 753.

[2] Briançon (Ch.), Thèse d'Etat, Orsay, mars, 1970.

[3] Sliv (L. A.), Band (I. M.), Gamma-Rays, Akad. Nauk., S. S. S. R., 1961.

[4] Church (E. L.), Weneser (J.), Phys. Rev., 1956, 103, $1035 ; 1956,104,1382$.
[5] Church (E. L.), Weneser (J.), Ann. Rev. of Nucl. Science, 1960, 10, 193.

[6] Herrlander (C. J.), Graham (R. L.), Nucl. Phys., 1964, 58, 544.

[7] Briançon (Ch.), Walen (R. J.), International Conference of Radioactivity, Montréal, 1969. 